\title{
Phase I study of liposomal irinotecan (LY01610) in patients with advanced esophageal squamous cell carcinoma
}

\author{
Yun Liu ${ }^{1} \cdot$ Bo Zhang ${ }^{1} \cdot$ Jianping X $\mathbf{u}^{1} \cdot$ Xingyuan Wang $^{1} \cdot$ Jialin Tang ${ }^{1} \cdot$ Jing Huang $^{1}(\mathbb{C}$ \\ Received: 20 January 2021 / Accepted: 15 May 2021 / Published online: 24 May 2021 \\ (c) The Author(s), under exclusive licence to Springer-Verlag GmbH Germany, part of Springer Nature 2021
}

\begin{abstract}
Purpose This phase I trial was performed to determine the maximum-tolerated dose (MTD), dose-limiting toxicities (DLTs), preliminary efficacy, and pharmacokinetics (PK) of LY01610, a novel liposome-encapsulated irinotecan, in patients with advanced esophageal squamous cell carcinoma (ESCC).

Methods This trial was conducted in two stages. In the dose-escalation stage, patients with advanced ESCC refractory or intolerant to previous chemotherapy received escalating doses of LY01610. A recommended dose based on patient tolerance was then expanded in the second stage. LY01610 was administered intravenously every 2 weeks, except that the first cycle in dose escalation was 3 weeks to allow observation of DLTs.

Results Twenty-four patients were enrolled across 4 dose levels $\left(30,60,90\right.$ and $\left.120 \mathrm{mg} / \mathrm{m}^{2}\right)$. The DLTs included vomiting and febrile neutropenia, and the MTD was $90 \mathrm{mg} / \mathrm{m}^{2}$. The most common grade $3 / 4$ adverse events were leukopenia in six patients $(25.0 \%)$, anemia in six patients $(25.0 \%)$ and neutropenia in five patients $(20.8 \%)$. One patient achieved complete response, and four had partial response, including one patient receiving LY01610 at the starting dose of $30 \mathrm{mg} / \mathrm{m}^{2}$. Compared with conventional irinotecan, the PK profile of LY01610 was characterized by increased and prolonged exposure of total irinotecan and the active metabolite $\mathrm{SN}-38$ in plasma.

Conclusion LY01610 demonstrated manageable toxicity and promising anti-tumor activity in patients with advanced ESCC. Future clinical development of LY01610 as single agent or in combination with other anti-cancer agents in treating ESCC patients is warranted.
\end{abstract}

Trial registration NCT04088604 at ClinicalTrials.gov.

\section{Introduction}

Esophageal cancer is the sixth driving cause of cancer-related death around the world [1, 2]. In Eastern Europe and Asia, esophageal squamous cell carcinoma (ESCC) remains the most prevalent histological subtype, accounting for roughly $90 \%$ of all esophageal cancer cases $[3,4]$. About two-thirds of patients with esophageal

Jianping $\mathrm{Xu}$ and Jing Huang contributed equally to this work.

Jianping Xu

13651379626@139.com

Jing Huang

huangjingwg@163.com

1 Department of Medical Oncology, National Cancer Center/National Clinical Research Center for Cancer/ Cancer Hospital, Chinese Academy of Medical Sciences and Peking Union Medical College, No.17 Panjiayuan Nanli, Chaoyang 100021, Beijing, China cancer are unresectable at the time of initial diagnosis, and $43.3-54.5 \%$ of patients undergoing radical esophagectomy have local recurrence or distant metastasis within 5 years $[5,6]$. Treatment options for patients with advanced ESCC are limited. Cisplatin combined with 5-fluorouracil is commonly used in the first-line setting. For ESCC patients whose disease has progressed with first-line therapy, irinotecan is among the preferred cytotoxic agents in the National Comprehensive Cancer Network (NCCN) Guidelines [7]. The response rate reported in a phase 2 trial with single-agent irinotecan in pretreated esophageal cancer patients was $15.4 \%[8]$.

Irinotecan is an inhibitor of DNA topoisomerase I. To be clinically effective, irinotecan must be converted to its active metabolite $\mathrm{SN}-38$ by carboxylesterase primarily in the liver. The mechanism of anti-tumor activity is through the binding of SN-38 to topoisomerase I-DNA complex, causing double-strand DNA damage during DNA synthesis [9]. SN-38 can subsequently be converted via glucuronosyl transferase 
1A1 (UGT1A1) conjugation to its inactive metabolite $\mathrm{SN}-38$ glucuronide (SN-38G). Upon irinotecan administration, biotransformation of SN-38 to SN-38G protects against gastrointestinal toxicity.

Liposomal irinotecan is an encapsulated nanoliposomal formulation of irinotecan hydrochloride. The new formulation protects the drug from premature conversion and activation in the liver, therefore the plasma circulation of liposomal irinotecan is prolonged [10]. It also enables the slow release of encapsulated drug to lower the maximum plasma concentration $\left(\mathrm{C}_{\max }\right)$ and to alleviate drug-associated side effects. Moreover, the leaky vasculature in tumor facilitates the extravasation of liposomal nanoparticles and the defective lymphatic drainage helps increase the retention of the drug within tumor, thereby enhancing its local accumulation in the tumor tissue [10-13]. Irinotecan liposome injection (Onivyde, Merrimack Pharmaceuticals; formerly PEP02 or MM-398) in combination with leucovorin (LV) and 5-fluorouracil (5-FU) has been approved by the FDA for the treatment of patients with metastatic pancreatic adenocarcinoma that relapsed after gemcitabine-based chemotherapy. However, no reports ever addressed treatment outcomes of liposomal irinotecan in patients with esophageal cancer.

LY01610 (Irinotecan hydrochloride liposome injection, Nanjing Luye Pharmaceutical Co., Ltd., Nanjing, China) is a novel nanoparticle formulation of irinotecan hydrochloride encapsulated with polyethylene glycolated liposome. Based on the efficacy of irinotecan in esophageal cancer patients in previous reports, and the presumed advantage of improved bioavailability and anti-tumor activity with LY01610 over conventional irinotecan, we performed a phase I study to evaluate the safety, pharmacokinetics and anti-tumor activity of LY01610 in treatment-refractory advanced ESCC patients.

\section{Patients and methods}

\section{Patient selection}

This is a phase I, open-label, non-randomized, dose escalation study of LY01610. Key inclusion criteria included: (i) having histologically confirmed solid tumors and have experienced disease progression or intolerance to standard systemic treatment; (ii) age $\geq 18$ and $\leq 70$ years; (iii) Eastern Cooperative Oncology Group performance status score $<2$; (iv) life expectancy $\geq 3$ months; (v) adequate bone marrow, liver and renal functions: absolute neutrophil count $\geq 1.5 \times 10^{9} / \mathrm{L}$, platelet count $\geq 100 \times 10^{9} / \mathrm{L}$ and hemoglobin $\geq 90 \mathrm{~g} / \mathrm{L}$; total bilirubin $\leq 1.5 \times$ upper limit of normal (ULN), alanine aminotransferase (ALT) and aspartate aminotransferase (AST) $\leq 2.5 \times$ ULN (ALT and $\mathrm{AST} \leq 5 \times \mathrm{ULN}$ for patients with liver metastasis); serum creatinine $\leq 1.5 \times \mathrm{ULN}$ or creatinine clearance rate $\geq 50 \mathrm{~mL} /$ min (Cockcroft-Gault formula); (vi) no chemotherapy or radiotherapy within 4 weeks before treatment initiation.

The exclusion criteria included: (i) active systemic infections requiring intravenous antibiotics; (ii) significant cardiovascular diseases, such as myocardial infarction, unstable angina, congestive heart failure (New York Heart Association $\geq$ class II), or unstable arrhythmia within 6 months before screening; (iii) uncontrolled ascites or pleural effusions; (iv) pregnancy, lactating or refusal to use effective contraception; (v) a second malignancy within 5 years prior to screening; (vi) symptomatic brain metastasis; (vii) previous exposure to irinotecan; (viii) UGT1A1 $* 28$ homozygous 7/7 variant.

The independent ethics committee approved all versions of the protocol and informed consent form (ICF). All participants voluntarily signed the written ICF. The study was conducted in compliance with the Declaration of Helsinki and the International Conference on Harmonization Good Clinical Practice guidelines. This trial was registered on ClinicalTrials.gov (Identifier: NCT04088604).

\section{Study design and treatments}

This study comprised of two stages: dose escalation and dose expansion. The starting dose of LY01610 was $30 \mathrm{mg} /$ $\mathrm{m}^{2}$, and the planned subsequent dose levels were 60,90 , 120,150 and $180 \mathrm{mg} / \mathrm{m}^{2}$. To effectively reduce the number of patients who are under-treated, dose escalation followed a modified patient cohort accelerated titration design [14], in which a single-patient cohort for $30 \mathrm{mg} / \mathrm{m}^{2}$ and three-patient cohorts for $60 \mathrm{mg} / \mathrm{m}^{2}$ or above would be recruited until any DLT was observed in the first circle. Once a patient experienced DLT, then additional patients would be enrolled at the same dose. Further dose escalation would be terminated if two or more of the patients experienced any DLT at a particular dose level, and the prior lower dose would be defined as the maximum-tolerated dose (MTD). Intra-patient dose escalation was not permitted. In the dose-expansion stage, 12-15 patients were enrolled to further evaluate the safety, PK characteristics and efficacy of LY01610. The dose for expansion was chosen based on the patient tolerance in dose escalation.

LY01610 was diluted in $250 \mathrm{~mL}$ of $5 \%$ dextrose and delivered as a 90-min intravenous infusion without premedication. In the dose-escalation stage, the first 3-week was defined as the observation period for DLTs. The treatment was then repeated every 2 weeks starting from the second dose until disease progression, unacceptable toxicity, treatment delay for $\geq 2$ weeks, or patient's refusal or death. In the dose-expansion stage, LY01610 was administered every 2 weeks. 


\section{Safety and response evaluation}

Adverse events (AEs) and DLTs were graded according to the National Cancer Institute Common Terminology Criteria for Adverse Events (NCI-CTCAE) version 5.0. DLT was defined as any of the following events occurring during the first 3-week period: (i) grade 4 neutropenia lasting for at least 3 days or grade 3 febrile neutropenia; (ii) grade 3 thrombocytopenia with bleeding; (iii) any other grade 4 hematologic or grade 3 non-hematologic toxicities. Grade 3 diarrhea, nausea, vomiting, or electrolyte disorders were considered DLT only when these disorders persistent despite appropriate medical management.

Radiographical studies were conducted every 8 weeks, and responses were assessed using the Response Evaluation Criteria in Solid Tumors (RECIST) version 1.1 [15].

\section{Pharmacokinetics}

Pharmacokinetic sampling was done during the first cycle of LY01610 administration. Blood samples were collected from an arm vein contralateral to the site of injection into $\mathrm{K}_{2}$-ethylenediaminetetraacetic acid (EDTA) Vacutainer tubes before treatment, during the infusion at $45 \mathrm{~min}$, at the end of infusion, after infusion at 1, 2, 3, 6, 9, 12, 24, 48, 72, 96, 168,240 and $336 \mathrm{~h}$, and before the second dose. Blood samples were kept at $4{ }^{\circ} \mathrm{C}$ while being processed for laboratory analyses. Plasma was separated by centrifugation within $1 \mathrm{~h}$ of blood collection. The original plasma samples were stored at $-80^{\circ} \mathrm{C}$ until batched analyses. Plasma levels of total irinotecan (encapsulated + free), free irinotecan, SN-38 and SN-38G were measured by validated liquid chromatography coupled to tandem mass spectrometry (LC/MS/MS) analytical methods. Irinotecan-d10 used as an internal standard for the quantification of free irinotecan was purchased from Toronto Research Chemicals (Toronto, ON, Canada). Separation of free irinotecan from liposomal was performed using Centrifree ${ }^{\circledR}$ Ultrafiltration Devices ( $1 \mathrm{~mL}$, Merck Millipore Ltd. Carrigtwohill, IRL). An aliquot of $50-\mu \mathrm{L}$ plasma sample marked by irinotecan-d10 was diluted with $1 \mathrm{~mL} 0.9 \%$ saline and transferred to the ultrafiltration device and centrifuged at $4{ }^{\circ} \mathrm{C}$ for $20 \mathrm{~min}$ at $2000 \mathrm{~g}$ in a centrifuge. A $200-\mu \mathrm{L}$ aliquot of the ultrafiltrate was processed for LC-MS/MS analysis. The average recovery rate reached $82.4 \%$ using quality control plasma samples at three different concentration level, and the coefficient of variation was $1.8 \%$, indicating that this method could effectively extract free irinotecan from plasma samples. In this trial, the assay used for sample detection had completed methodological verification and was consistent with FDA and European Medicines Agency (EMA) guidance. Study samples were reanalyzed and matched incurred sample reanalysis (ISR) criteria with a $100 \%$ pass rate. The lower limits of quantitation for total irinotecan, free irinotecan, SN-38 and SN-38G were $100 \mathrm{ng} / \mathrm{mL}, 1 \mathrm{ng} / \mathrm{mL}$, $0.2 \mathrm{ng} / \mathrm{mL}$ and $1 \mathrm{ng} / \mathrm{mL}$, respectively.

PK parameters were calculated by a noncompartmental method using the Phoenix WinNonlin software (Version 8.1.0; Certara USA, Inc.; Princeton, NJ). Maximum concentration $\left(\mathrm{C}_{\max }\right)$ and time to reach $\mathrm{C}_{\max }\left(\mathrm{T}_{\max }\right)$ were taken directly from the observed data. Area under the plasma concentration-time curve from time zero to the time of the last observable concentration $\left(\mathrm{AUC}_{0 \rightarrow \mathrm{t}}\right)$ was determined by the linear-up/log-down trapezoidal rule. The $\mathrm{AUC}_{\text {inf }}$ was extrapolated from $\mathrm{AUC}_{0 \rightarrow \mathrm{t}}$ upon the observed last concentration using the Phoenix WinNonlin software. Phoenix first attempted to estimate the rate constant, Lambda $Z\left(\lambda_{Z}\right)$, associated with the terminal elimination phase for concentration data. If $\lambda_{Z}$ was estimable, parameters for concentration data would be extrapolated to infinity. To estimate the best fit for $\lambda_{Z}$, Phoenix repeats regressions of the natural logarithm of the concentration values using the last three points with non-zero concentrations, then the last four points, last five points, etc. The point at $C_{\max }$ for intravenous infusion model was not used in the Best Fit method. For each regression, $\mathrm{R}^{2}$ was computed in Supplementary Table 1 . The proportion of AUC from observed last concentration to infinity was shown in Supplementary Table 2.

\section{Endpoints and statistical analysis}

The primary endpoints were the MTD, DLT and safety profile of LY01610. Secondary endpoints were PK, objective response rate (ORR, defined as the percentage of patients whose best overall response was complete or partial response), disease control rate (DCR, defined as the percentage of patients whose best overall response was complete response, partial response, or stable disease), progressionfree survival (PFS, defined as the time from treatment initiation to the first disease progression or death from any cause), and overall survival (OS, defined as the time from treatment initiation to death from any cause).

The analyses were descriptive. Categorical variables were summarized as frequency and percentage, and continuous variables were summarized as mean with standard deviation (SD) or median with range. The Kaplan-Meier method was used to estimate time-to-event variables. All analyses were carried out on SPSS statistics 26 (International Business Machines (IBM) Corp., USA) and Phoenix WinNonlin software.

\section{Results}

\section{Patient characteristics}

Between February 2019 and August 2020, 34 patients were screened for this trial and 24 patients with advanced ESCC 
were eligible, with 13 in the dose-escalation stage and 11 in the dose-expansion stage. The median age was 59.0 years (range: 44-70), and 22 of the patients (91.7\%) were men. All patients had received at least one systemic therapy before treatment with LY01610. Apart from chemotherapy, other prior treatments included surgical resection $(n=10,41.7 \%)$ and radiotherapy $(n=14,58.3 \%)$. The demographics and baseline characteristics of all patients are listed in Table 1. Of note, this study was suspended from January 31, 2020 to March 6, 2020 due to the COVID-19 pandemic in China. As a result, two patients did not receive the planed treatment during the study suspension. One patient had disease progression after the treatment interruption, and another patient missed two cycles of the study treatment before resuming LY01610. The median follow-up duration was 13.1 months (95\% CI 11.3-14.8) as of the data cut-off date (October 7, 2020), and all patients had discontinued the study treatment. The reasons for treatment discontinuation were progressive disease $(n=19 ; 79.2 \%)$, adverse event $(n=1 ; 4.2 \%)$, patient refusal $(n=1 ; 4.2 \%)$, and death $(n=3 ; 12.5 \%)$.

Table 1 Demographic characteristics

\begin{tabular}{ll}
\hline & $N(\%)$ \\
\hline Patients enrolled & 24 \\
Age [median (range)] & $59(40-77)$ \\
Gender & \\
Male & $22(91.7)$ \\
Female & $2(8.3)$ \\
ECOG PS score & \\
0 & $20(83.3)$ \\
1 & $4(16.7)$ \\
Histologic grade & \\
G1 & $1(4.2)$ \\
G2 & $11(45.8)$ \\
G3 & $8(33.3)$ \\
Unknown & $4(16.7)$ \\
Number of organs with metastasis & \\
$\leq 1$ & $13(54.2)$ \\
$\geq 2$ & $11(45.8)$ \\
Site of metastases & \\
Lymph node & $19(79.2)$ \\
Lung & $7(29.2)$ \\
Liver & $7(29.2)$ \\
Bone & $2(8.3)$ \\
Surgery & $10(41.7)$ \\
Radiotherapy & $14(58.3)$ \\
1 & \\
2 & $21(87.5)$ \\
\hline & $3(12.5)$ \\
\hline Number of previous chemotherapy regimens & \\
& \\
&
\end{tabular}

ECOG Eastern Cooperative Oncology Group, $P S$ performance status

\section{DLT, MTD and safety}

Four dose levels were evaluated in the dose-escalation stage, with 2, 3, 6 and 2 patients enrolled in the 30, 60, 90 and $120 \mathrm{mg} / \mathrm{m}^{2}$ groups, respectively. One patient in the $30 \mathrm{mg} /$ $\mathrm{m}^{2}$ group died of upper gastrointestinal hemorrhage due to primary tumor invasion during the DLT observation period, and a second patient was enrolled at the same dose. No DLTs were observed at the 30 and $60 \mathrm{mg} / \mathrm{m}^{2}$ dose groups. One patient in the $90 \mathrm{mg} / \mathrm{m}^{2}$ group developed grade 3 febrile neutropenia, and a total of six patients were enrolled at this dose level, with no additional DLTs observed. Two patients in the $120 \mathrm{mg} / \mathrm{m}^{2}$ group developed grade 3 vomiting that did not improve after appropriate supportive therapy. Therefore, $90 \mathrm{mg} / \mathrm{m}^{2}$ was determined as the MTD of LY01610. In the dose-expansion stage, we enrolled 11 additional patients at $60 \mathrm{mg} / \mathrm{m}^{2}$ based on the safety findings in dose escalation.

Among the 24 patients who had received at least 1 dose of LY01610, the total cycles of LY01610 administered were 114, with a median of 5 cycles per patient (range: 1-17). Treatment delay and dose modification in each dose level are summarized in Table 2. The most frequent treatment-related AEs (TRAEs) were fatigue (100.0\%), anorexia (91.7\%), and nausea $(87.5 \%)$, and the majority of these TRAEs were of grade $1-2$. Without preventive atropine administration, no patients experienced cholinergic syndrome throughout the study. Treatment-related serious AEs were reported in four patients (16.7\%), including one case of grade 4 febrile neutropenia along with grade 4 leukopenia and grade 4 neutropenia, one case of grade 3 febrile neutropenia, and two cases of grade 3 vomiting. All TRAEs were managed with appropriate medical care. There were no treatment-related deaths. TRAEs observed in patients treated with LY01610 are listed in Table 3.

The frequency and grade of TRAEs were dose related. As dose escalation proceeded, hematologic toxicity became the most common grade $\geq 3$ AEs, among which leucopenia and neutropenia were more frequent at higher dose levels, whereas thrombocytopenia was uncommon across all dose levels. LY01610 was infused without premedication in this phase I study. Four patients experienced transient flushing, palpitation, and chest tightness at the beginning of first infusion, then the symptoms were relieved by reducing the infusion rate immediately. No infusion reactions recurred in these patients in the subsequent cycles.

\section{Antitumor activity}

Twenty-one of the 24 patients treated with LY01610 were evaluable for response. The ORR was $20.8 \%$, and the DCR was $33.3 \%$. One patient had a best response of complete response (CR) $(4.2 \%)$, four patients had partial response (PR) (16.6\%), and three (12.5\%) had stable disease (SD) (Fig. 1). 


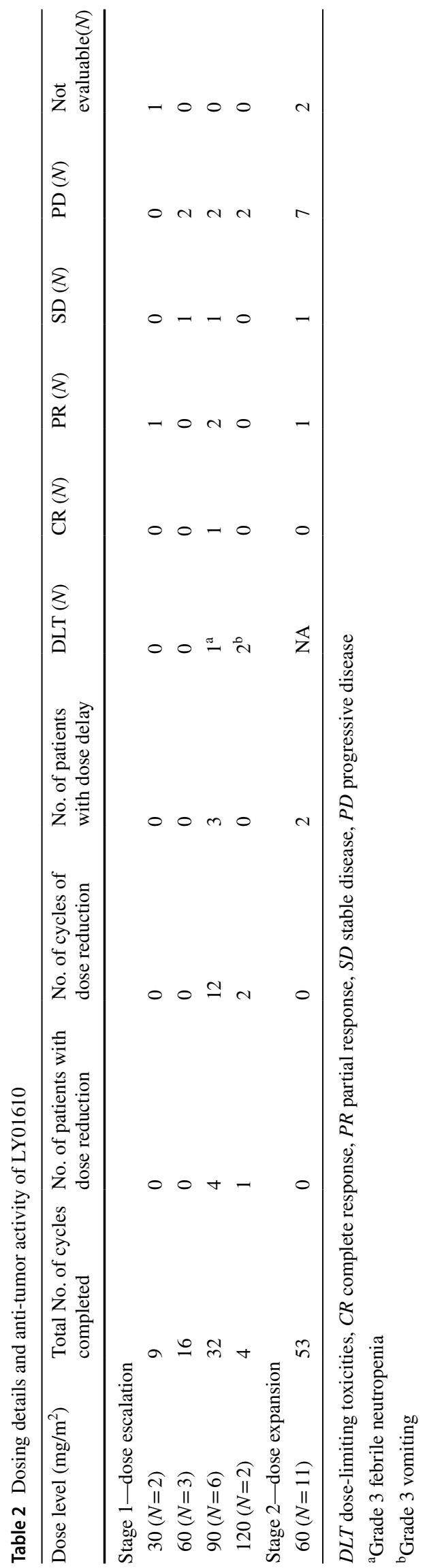

Among the five patients achieving objective responses, one received LY01610 at $30 \mathrm{mg} / \mathrm{m}^{2}$, one at $60 \mathrm{mg} / \mathrm{m}^{2}$, and three at $90 \mathrm{mg} / \mathrm{m}^{2}$. The response to LY01610 of each patient is summarized in Table 2. Median PFS (95.8\% maturity, 23 events from 24 patients) was 1.9 months (95\% CI: 1.7-2.1), and median OS (62.5\% maturity, 15 deaths from 24 patients) was 6.8 months (95\% CI: 3.9-9.7). Three patients were not assessed for response due to deterioration of cancer-related symptoms before the planned first imaging evaluation. They discontinued the study treatment afterwards and received supportive care.

\section{Pharmacokinetics}

The $\mathrm{C}_{\max }, \mathrm{AUC}_{0 \rightarrow t}, \mathrm{AUC}_{\mathrm{inf}}, \mathrm{T}_{1 / 2}$ and $\mathrm{T}_{\max }$ of total irinotecan, free irinotecan, $\mathrm{SN}-38$ and $\mathrm{SN}-38 \mathrm{G}$ at different dose levels are listed in Table 4 . We used the power function model to analyze the dose linearity of total irinotecan, free irinotecan, $\mathrm{SN}-38$ and SN-38G. The $\mathrm{C}_{\max }, \mathrm{AUC}_{0 \rightarrow \mathrm{t}}$ and $\mathrm{AUC}_{\text {inf }}$ of total irinotecan showed good linearity within the dose range of $30-120 \mathrm{mg} / \mathrm{m}^{2}$, with all $\mathrm{R}^{2}$ of the linear regression curves greater than 0.97 (shown in Supplementary Fig. 1). On the other hand, due to the small number of patients and inter-individual variation, the PK linearity across the entire range for free irinotecan, SN-38 and SN-38G were unable to be determined. But higher AUC was observed at higher dose levels.

The mean plasma concentration-time curve with positive SDs of total irinotecan, free irinotecan, SN-38 and SN38G at different dose levels are graphed in Fig. 2. LY01610 mainly presented in the plasma in an encapsulated form after infusion, with extremely low levels of free irinotecan (accounts for about $0.5-0.8 \%$ of total irinotecan) detected at different timepoints in the circulation. These results suggest the slow release of irinotecan from LY01610 over time.

\section{Discussion}

Irinotecan obtained approval for the treatment of cancer over 20 years ago. Up till now, successful development of a liposomal form of irinotecan with reduced toxicity and improved efficacy have been few. Onivyde (formerly PEP02 or MM-398) was the only marketed liposomal formulation, approved in combination with fluorouracil and folinic acid in patients with metastatic pancreatic adenocarcinoma who previously received gemcitabine-based therapy. IHL-305, another pegylated liposomal irinotecan, showed relatively low SN-38 AUC in its MTD on a 2-week dosing schedule [16]. The anti-tumor activity was limited, with only 1 case of PR observed in 60 enrolled patients, and further clinical development was terminated. In our present first-in-human study, we evaluated the safety, PK and preliminary efficacy 


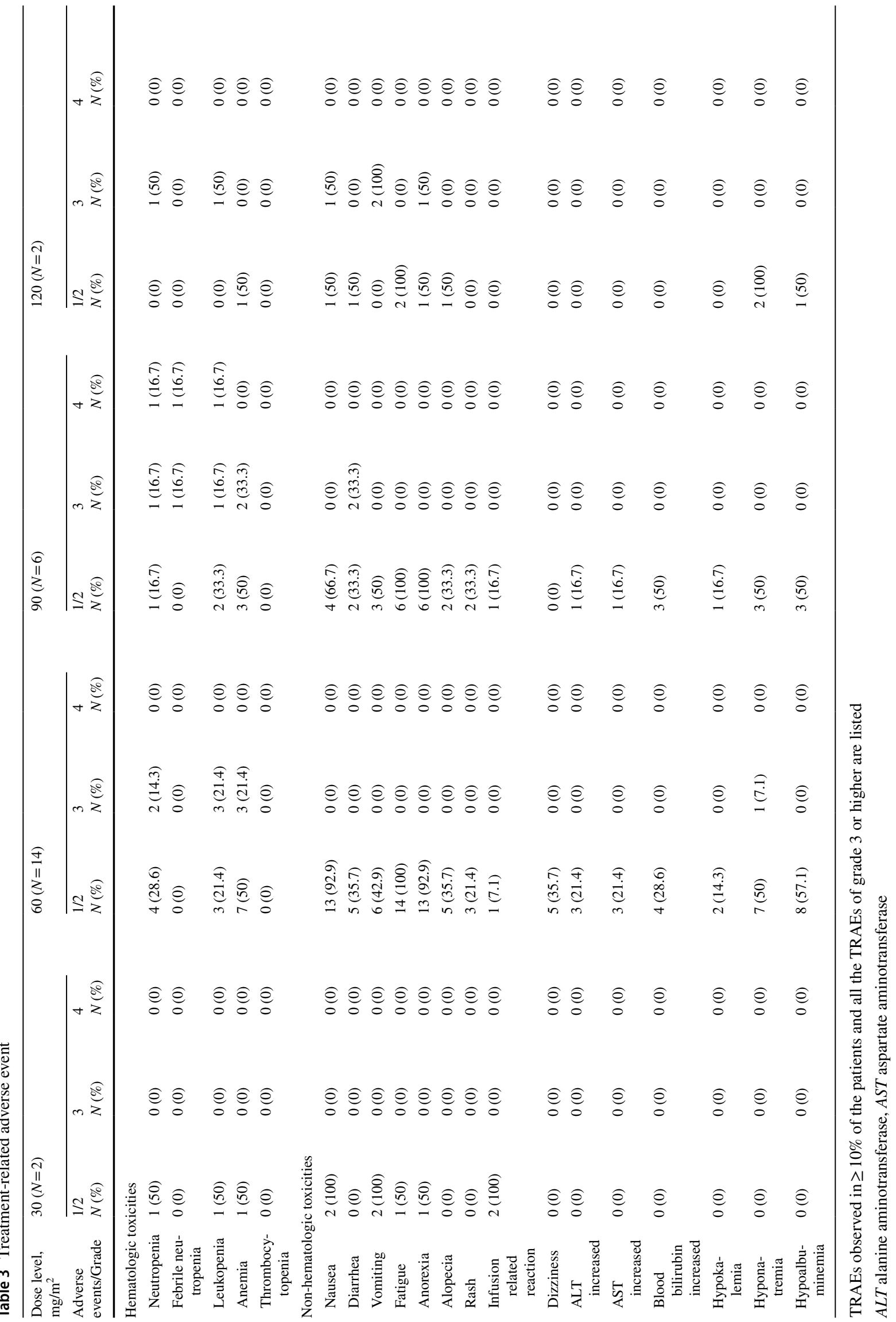



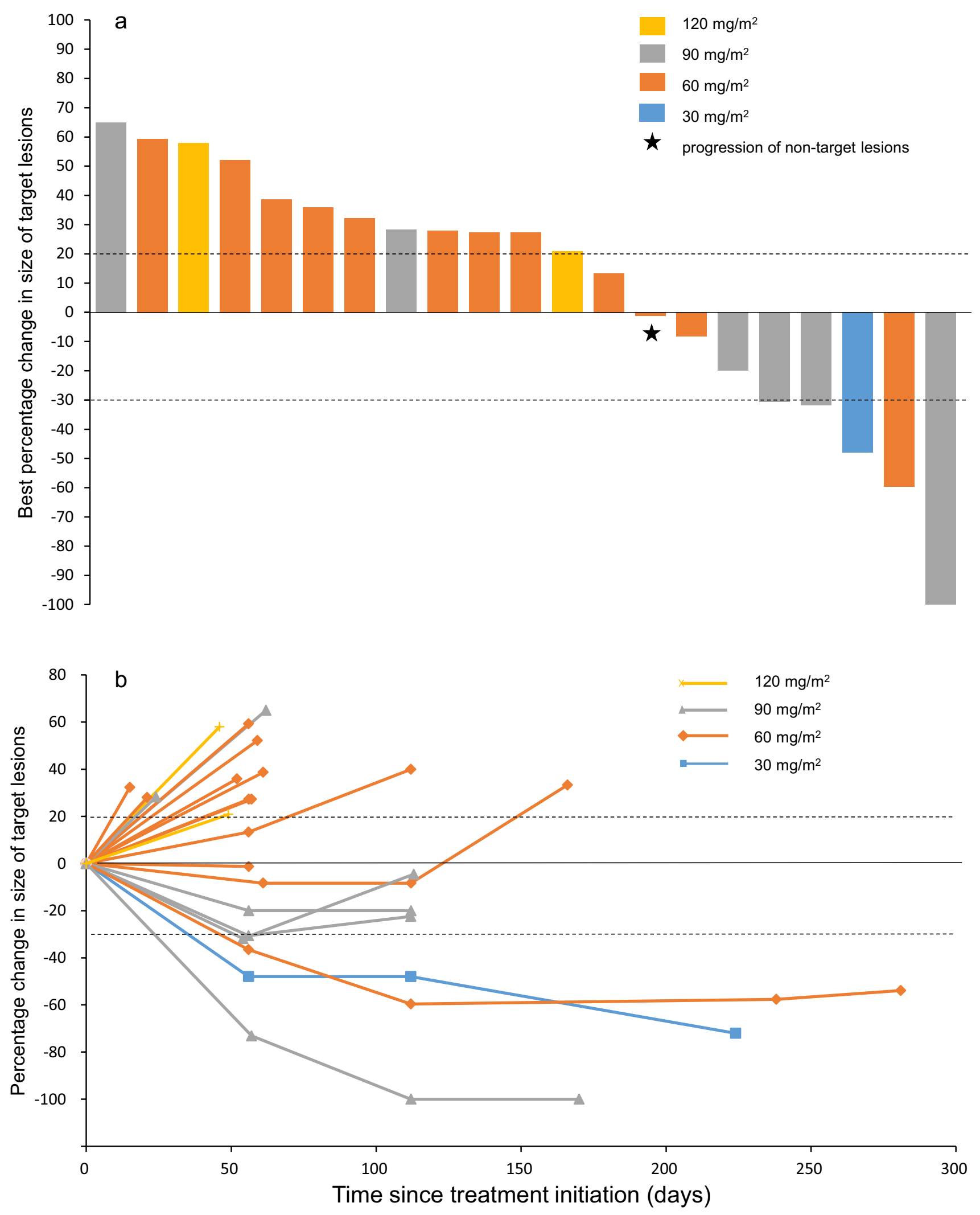

Fig. 1 Response to the study treatment. a Waterfall plot of best percent change in target lesions from baseline. The star indicates a patient with a new lesion despite stable target lesions. b Percentage change of target lesions from baseline 
Table 4 Pharmacokinetic parameters of LY01610 at each dose level

\begin{tabular}{|c|c|c|c|c|c|}
\hline \multirow[t]{2}{*}{ Dose level $\left(\mathrm{mg} / \mathrm{m}^{2}\right)$} & \multicolumn{5}{|l|}{ Mean (SD) } \\
\hline & $\mathrm{C}_{\max }(\mathrm{ng} / \mathrm{mL})$ & $\mathrm{AUC}_{0 \sim \mathrm{t}}(\mathrm{ng} / \mathrm{mL} \times \mathrm{h})$ & $\mathrm{AUC}_{\mathrm{inf}}(\mathrm{ng} / \mathrm{mL} \times \mathrm{h})$ & $\mathrm{t}_{1 / 2}(\mathrm{~h})$ & $\mathrm{T}_{\max }{ }^{\mathrm{a}}(\mathrm{h})$ \\
\hline \multicolumn{6}{|l|}{ Total irinotecan } \\
\hline $30 \mathrm{mg} / \mathrm{m}^{2}(n=2)$ & $14,451.58(2162.03)$ & $316,593.99(45,555.62)$ & $319,173.23(44,619.65)$ & $11.70(1.48)$ & 3.5 \\
\hline $60 \mathrm{mg} / \mathrm{m}^{2}(n=14)$ & $28,461.74(7405.29)$ & $611,697.63(214,012.85)$ & $615,722.68(215,128.93)$ & $11.75(2.47)$ & 1.5 \\
\hline $90 \mathrm{mg} / \mathrm{m}^{2}(n=6)$ & $48,806.27(6167.02)$ & $1,298,712.05(204,104.06)$ & $1,314,436.08(210,911.72)$ & $14.25(1.24)$ & 2.5 \\
\hline $120 \mathrm{mg} / \mathrm{m}^{2}(n=2)$ & $71,663.25(8156.77)$ & $1,497,515.80(516,081.32)$ & $1,507,701.25(524,511.66)$ & $13.32(1.35)$ & 3 \\
\hline \multicolumn{6}{|l|}{ Free irinotecan } \\
\hline $30 \mathrm{mg} / \mathrm{m}^{2}(n=2)$ & $33.86(4.59)$ & $1639.52(13.64)$ & $1723.52(16.15)$ & $19.35(1.88)$ & 15 \\
\hline $60 \mathrm{mg} / \mathrm{m}^{2}(n=14)$ & $97.62(41.00)$ & $4916.26(1940.08)$ & $5034.00(2034.16)$ & $33.18(18.42)$ & 25.5 \\
\hline $90 \mathrm{mg} / \mathrm{m}^{2}(n=6)$ & $105.41(43.95)$ & $6250.23(1762.89)$ & $6322.10(2087.52)$ & $25.44(1.42)$ & 25.4 \\
\hline $120 \mathrm{mg} / \mathrm{m}^{2}(n=2)$ & 211.47 (72.19) & $8673.69(5262.09)$ & $8825.92(5403.62)$ & $27.10(5.18)$ & 7.5 \\
\hline \multicolumn{6}{|l|}{ SN-38 } \\
\hline $30 \mathrm{mg} / \mathrm{m}^{2}(n=2)$ & $2.71(0.91)$ & $147.97(57.80)$ & $171.35(76.68)$ & $25.86(4.45)$ & 10.5 \\
\hline $60 \mathrm{mg} / \mathrm{m}^{2}(n=14)$ & $6.44(3.29)$ & $426.54(281.81)$ & $455.42(286.11)$ & $42.93(11.72)$ & 13.5 \\
\hline $90 \mathrm{mg} / \mathrm{m}^{2}(n=6)$ & $6.45(4.09)$ & $458.96(239.81)$ & $475.81(238.99)$ & $39.45(4.05)$ & 25.5 \\
\hline $120 \mathrm{mg} / \mathrm{m}^{2}(n=2)$ & $11.05(6.48)$ & $529.33(457.73)$ & $553.01(452.53)$ & $53.57(8.60)$ & 19.5 \\
\hline \multicolumn{6}{|l|}{ SN-38G } \\
\hline $30 \mathrm{mg} / \mathrm{m}^{2}(n=2)$ & $7.35(1.28)$ & $479.19(102.87)$ & 458.19 (NA) & $24.82(\mathrm{NA})$ & 37.5 \\
\hline $60 \mathrm{mg} / \mathrm{m}^{2}(n=14)$ & $28.55(16.72)$ & 1993.81(1397.08) & 2109.76(1439.28) & $36.15(8.77)$ & 25.5 \\
\hline $90 \mathrm{mg} / \mathrm{m}^{2}(n=6)$ & $18.5(5.78)$ & $1332.62(445.13)$ & $1467.02(443.59)$ & $35.19(3.34)$ & 25.5 \\
\hline $120 \mathrm{mg} / \mathrm{m}^{2}(n=2)$ & $38.71(8.44)$ & $2415.88(186.16)$ & $2601.83(26.06)$ & $48.19(0.42)$ & 16.5 \\
\hline
\end{tabular}

NA: one of the patients had less than three measurable plasma samples after $\mathrm{T}_{\max }$, which could not fit the elimination parameters of $\mathrm{AUC}_{\mathrm{inf}}$ and $t_{1 / 2}$

${ }^{\mathrm{a}} \mathrm{T}_{\max }$ is represented by the median

of LY01610, a novel nanoparticle formulation of irinotecan, in patients with advanced ESCC. The safety profile of LY01610 administered every 2 weeks was favorable. Myelosuppression and gastrointestinal events were the major DLTs, and $90 \mathrm{mg} / \mathrm{m}^{2}$ was defined as the MTD. The PK results suggested a slow but durable release of total irinotecan and SN-38. In addition, LY01610 was active in patients with pretreated advanced ESCC, with 1 case of CR and 4 cases of PR among the 24 enrolled patients. Although LY01610 was developed as an analog of Onivyde, the category and proportion of phospholipid vesicle (liposome) encapsulating irinotecan hydrochloride were different. Besides, the distinctive preparation technology and quality control standards may contribute to the different PK characteristics. To our knowledge, this is the first report on the treatment outcomes and $\mathrm{PK}$ parameters of a liposomal irinotecan in patients with advanced ESCC, the differences of clinical efficacy between LY01610 and Onivyde were equivocal and warranted further clinical investigations.

No new safety signals were identified with this novel liposomal formulation, and the AEs observed in the present trial were comparable to those with Onivyde [17]. Treatment-related adverse events were generally manageable with appropriate supportive care or dose interruptions or reductions, indicating that LY01610 was well tolerated in patients with ESCC. Additionally, we did not administer prophylactic atropine before LY01610 dosing, and no cholinergic reactions were observed throughout the study. This was consistent with the findings in the phase 1 study of Onivyde [17]. In contrast, the reported incidence of overall irinotecan-related cholinergic syndrome ranged from 31.3 to $83.0 \%$ in patients receiving irinotecan-based chemotherapies [18-20]. The patients in the present trial might have benefited from the lower $\mathrm{C}_{\max }$ of free irinotecan, since the frequency and severity of cholinergic syndrome are likely irinotecan concentration dependent [21, 22].

Irinotecan was commonly used at $180 \mathrm{mg} / \mathrm{m}^{2}$ every 2 weeks as monotherapy or in combination with other cytotoxic drugs in treating gastrointestinal cancers. Comparing the PK of SN-38 in our present study with that from a phase 1 study evaluating conventional irinotecan at $180 \mathrm{mg} /$ $\mathrm{m}^{2}, \mathrm{C}_{\max }$ was significantly lowered with LY01610 at the MTD $\left(90 \mathrm{mg} / \mathrm{m}^{2}\right.$ ) (Mean: $6.45 \mathrm{vs} 26.2 \mathrm{ng} / \mathrm{mL}$ ), meanwhile, longer $\mathrm{T}_{1 / 2}(39.45 \mathrm{vs} 19.7 \mathrm{~h})$ and higher $\mathrm{AUC}_{\text {inf }}(475.81 \mathrm{vs}$ $367.6 \mathrm{ng} / \mathrm{mL}^{*} \mathrm{~h}$ ) were observed [23]. Notably, the $\mathrm{AUC}_{\text {inf }}$ of SN-38 with LY01610 at $60 \mathrm{mg} / \mathrm{m}^{2}(455.42 \mathrm{ng} / \mathrm{mL} * \mathrm{~h}$, listed in Table 4) was already higher than that achieved with $180 \mathrm{mg} / \mathrm{m}^{2}$ conventional irinotecan, suggesting equivalent or 

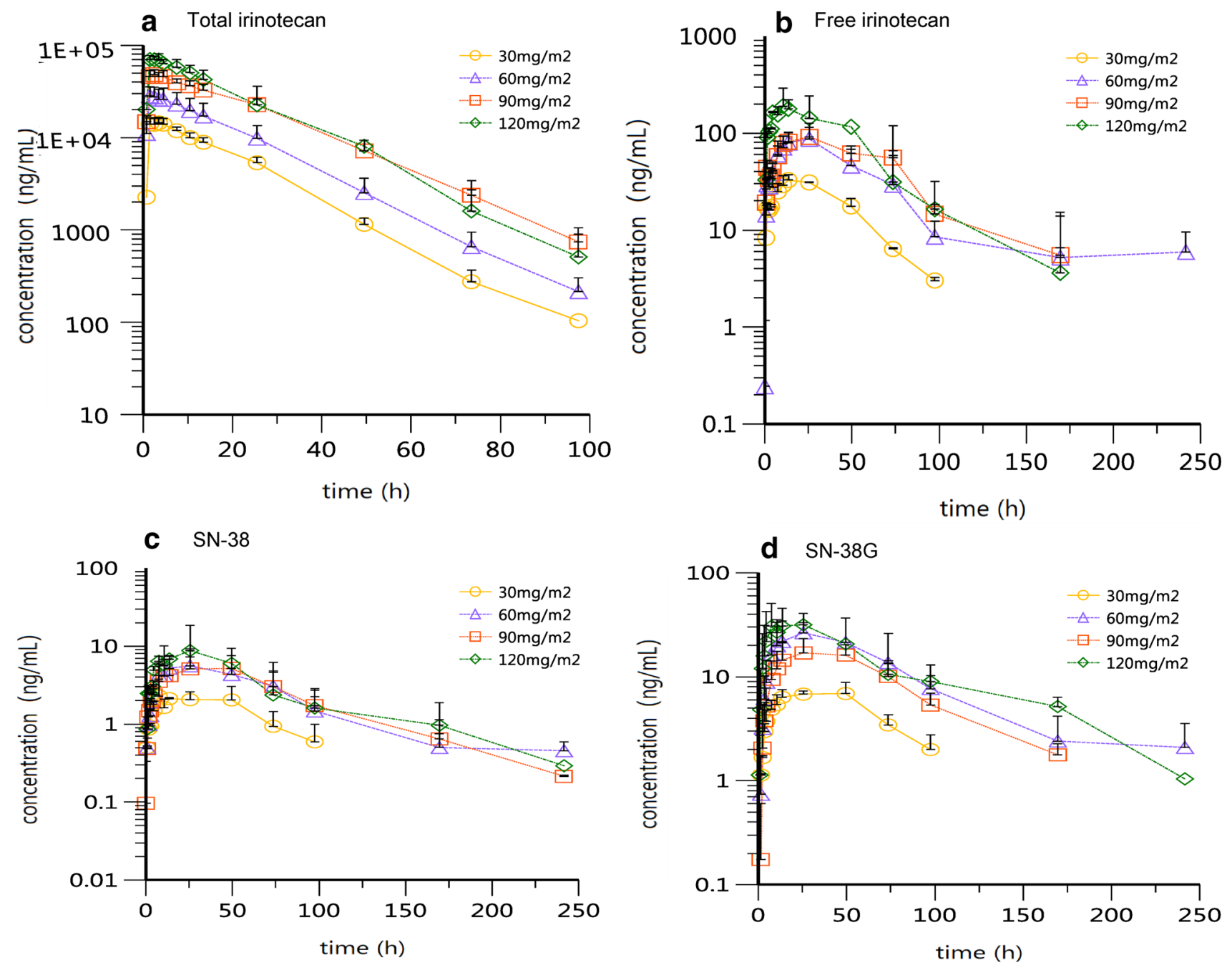

Fig. 2 Mean plasma concentration-time curve with positive standard deviations (SDs). a Total irinotecan, b free irinotecan, c SN-38 and d SN$38 \mathrm{G}$ at 30, 60, 90 and $120 \mathrm{mg} / \mathrm{m} 2$ dose levels of LY01610

improved efficacy with lower toxicities. Indeed, comparing the safety profile with single-agent irinotecan at $180 \mathrm{mg} /$ $\mathrm{m}^{2}$ administered every 14 days in a phase 2 clinical trial, LY01610 at $60 \mathrm{mg} / \mathrm{m}^{2}$ was better tolerated with a numerically lower incidence of grade 3 or 4 neutropenia ( $14.3 \%$ vs $23.3 \%$ ) and thrombocytopenia (0\% vs $3.3 \%$ ), although the differences were not statistically tested [24].

Currently, evidence of the anti-tumor activity of singleagent irinotecan in patients with advanced ESCC was limited. In a phase 2 study including both patients with pretreated ESCC and esophageal adenocarcinoma, the response rate of weekly irinotecan monotherapy was $15 \%$, and the median PFS and OS were 2 months and 5 months, respectively [8]. Recently, the ESCORT study compared camrelizumab (a PD-1 inhibitor) with investigator's choice of chemotherapy in patients with advanced ESCC, in which the investigator's choice of chemotherapy included irinotecan and docetaxel. In the chemotherapy arm, 80.5\% (177/220) patients received irinotecan monotherapy, and the reported ORR was $6.4 \%$ and the median OS was 6.2 months (95\% CI: 5.7-6.9). Taking as reference the response rates in these two prior trials, the efficacy of LY01610 in the present study was encouraging, with an ORR of $20.8 \%$. The median PFS and OS observed in our present study (1.9 months and 6.8 months, respectively) were similar to the survival data in previous trials. However, it is noteworthy that the suspension of the current study during the COVID-19 pandemic in early 2020 might have caused an underestimation of the PFS, since two patients who responded to LY01610 missed the scheduled study treatment during the suspension. Besides, one patient having a best response of PR received LY01610 at the starting dose of $30 \mathrm{mg} / \mathrm{m}^{2}$. The potential of LY01610 to achieve robust anti-tumor activity at a dose level significantly lower than the MTD suggested a wide therapeutic 
index. This is clinically meaningful in that a lower dose level might be used in the setting of maintenance therapy until disease progression, or unacceptable toxicity in the future.

In the era of immunotherapy, three randomized phase 3 trials with immune checkpoint inhibitors have established anti-PD-1 antibodies as the standard second-line regimen for advanced ESCC, and thus changed the management of this devastating malignancy [25-27]. However, the benefit in response and survival with single-agent anti-PD-1 antibodies in an unselected patient population was limited, and chemotherapy was therefore still of important value. In the KEYNOTE-590 trial, an improvement in OS was observed in ESCC patients treated with pembrolizumab plus chemotherapy versus chemotherapy alone as first-line therapy, validating the promise of this combination strategy in ESCC [28]. To achieve better treatment outcomes in the future, the optimal chemotherapy backbone in combination with immunotherapy remained to be investigated. Since irinotecan is among the effective cytotoxic agents against ESCC, the antitumor activity observed with LY01610 in our present trial and the improved tolerance support future clinical development this novel agent in combination with other cytotoxic drugs and/or PD-1 inhibitors in ESCC patients.

In conclusion, LY01610 was safe and effective in patients with advanced ESCC refractory or intolerant to previous chemotherapy in our phase 1 study, with slow but prolonged release of total irinotecan and $\mathrm{SN}-38$ as revealed by PK studies. LY01610 warrants further validation in randomized trials as single agent or in combination with other anti-cancer agents in treating ESCC patients.

Supplementary Information The online version contains supplementary material available at https://doi.org/10.1007/s00280-021-04294-2.

Acknowledgements We thank Jing Tong for performing the pharmacokinetic analysis of this study. We thank the patients, their families, and the study personnel involved in this trial.

Funding The study was funded by Nanjing Luye Pharmaceutical Co., Ltd., Nanjing, China.

Availability of data and materials Data supporting the results reported in this article are not publicly available but are accessible from the corresponding author on reasonable request and approval from study sponsor according to available guidelines at time of request.

\section{Declarations}

Conflict of interest The author(s) declare that they have no conflict of interest.

Ethics approval and consent to participate This study was conducted in accordance with the principles of the Declaration of Helsinki and the International Conference on Harmonization Guidelines for Good Clinical Practice. The study protocol was approved by the ethics committee. Informed consent was obtained from all individual participants included in the study.

\section{References}

1. Fitzmaurice C, Allen C, Barber RM, Barregard L, Bhutta ZA, Brenner H, Dicker DJ, Chimed-Orchir O, Dandona R, Dandona L, Fleming T, Forouzanfar MH, Hancock J, Hay RJ, Hunter-Merrill R, Huynh C, Hosgood HD, Johnson CO, Jonas JB, Khubchandani J, Kumar GA, Kutz M, Lan Q, Larson HJ, Liang X, Lim SS, Lopez AD, MacIntyre MF, Marczak L, Marquez N, Mokdad AH, Pinho C, Pourmalek F, Salomon JA, Sanabria JR, Sandar L, Sartorius B, Schwartz SM, Shackelford KA, Shibuya K, Stanaway J, Steiner C, Sun J, Takahashi K, Vollset SE, Vos T, Wagner JA, Wang H, Westerman R, Zeeb H, Zoeckler L, Abd-Allah F, Ahmed MB, Alabed S, Alam NK, Aldhahri SF, Alem G, Alemayohu MA, Ali R, Al-Raddadi R, Amare A, Amoako Y, Artaman A, Asayesh H, Atnafu N, Awasthi A, Saleem HB, Barac A, Bedi N, Bensenor I, Berhane A, Bernabe E, Betsu B, Binagwaho A, Boneya D, Campos-Nonato I, Castaneda-Orjuela C, Catala-Lopez F, Chiang P, Chibueze C, Chitheer A, Choi JY, Cowie B, Damtew S, das Neves J, Dey S, Dharmaratne S, Dhillon P, Ding E, Driscoll T, Ekwueme D, Endries AY, Farvid M, Farzadfar F, Fernandes J, Fischer F, TT GH, Gebru A, Gopalani S, Hailu A, Horino M, Horita N, Husseini A, Huybrechts I, Inoue M, Islami F, Jakovljevic M, James S, Javanbakht M, Jee SH, Kasaeian A, Kedir MS, Khader YS, Khang YH, Kim D, Leigh J, Linn S, Lunevicius R, El Razek HMA, Malekzadeh R, Malta DC, Marcenes W, Markos D, Melaku YA, Meles KG, Mendoza W, Mengiste DT, Meretoja TJ, Miller TR, Mohammad KA, Mohammadi A, Mohammed S, Moradi-Lakeh M, Nagel G, Nand D, Le Nguyen Q, Nolte S, Ogbo FA, Oladimeji KE, Oren E, Pa M, Park EK, Pereira DM, Plass D, Qorbani M, Radfar A, Rafay A, Rahman M, Rana SM, Soreide K, Satpathy M, Sawhney M, Sepanlou SG, Shaikh MA, She J, Shiue I, Shore HR, Shrime MG, So S, Soneji S, Stathopoulou V, Stroumpoulis K, Sufiyan MB, Sykes BL, Tabares-Seisdedos R, Tadese F, Tedla BA, Tessema GA, Thakur JS, Tran BX, Ukwaja KN, Uzochukwu BSC, Vlassov VV, Weiderpass E, Wubshet Terefe M, Yebyo HG, Yimam HH, Yonemoto N, Younis MZ, Yu C, Zaidi Z, Zaki MES, Zenebe ZM, Murray CJL, Naghavi M,, Global Burden of Disease Cancer C (2017) Global, regional, and national cancer incidence, mortality, years of life lost, years lived with disability, and disability-adjusted life-years for 32 cancer groups, 1990 to 2015: a systematic analysis for the global burden of disease study. JAMA oncol 3(4):524-548. https://doi.org/10. 1001/jamaoncol.2016.5688

2. Bray F, Ferlay J, Soerjomataram I, Siegel RL, Torre LA, Jemal A (2018) Global cancer statistics 2018: GLOBOCAN estimates of incidence and mortality worldwide for 36 cancers in 185 countries. CA: Cancer J Clinic 68(6):394-424. https://doi.org/10.3322/ caac. 21492

3. Cancer Genome Atlas Research N, Analysis Working Group: Asan U, Agency BCC, Brigham, Women's H, Broad I, Brown U, Case Western Reserve U, Dana-Farber Cancer I, Duke U, Greater Poland Cancer C, Harvard Medical S, Institute for Systems B, Leuven KU, Mayo C, Memorial Sloan Kettering Cancer C, National Cancer I, Nationwide Children's H, Stanford U, University of A, University of M, University of North C, University of P, University of R, University of Southern C, University of Texas MDACC, University of W, Van Andel Research I, Vanderbilt U, Washington U, Genome Sequencing Center: Broad I, Washington University in St L, Genome Characterization Centers BCCA, Broad I, Harvard Medical S, Sidney Kimmel Comprehensive Cancer Center at Johns Hopkins U, University of North C, University of Southern California Epigenome C, University of Texas MDACC, Van Andel Research I, Genome Data Analysis Centers: Broad I, Brown U, Harvard Medical S, Institute for Systems B, Memorial Sloan Kettering Cancer C, University of 
California Santa C, University of Texas MDACC, Biospecimen Core Resource: International Genomics C, Research Institute at Nationwide Children's H, Tissue Source Sites: Analytic Biologic S, Asan Medical C, Asterand B, Barretos Cancer H, BioreclamationIvt, Botkin Municipal C, Chonnam National University Medical S, Christiana Care Health S, Cureline, Duke U, Emory U, Erasmus U, Indiana University School of M, Institute of Oncology of M, International Genomics C, Invidumed, Israelitisches Krankenhaus H, Keimyung University School of M, Memorial Sloan Kettering Cancer C, National Cancer Center G, Ontario Tumour B, Peter MacCallum Cancer C, Pusan National University Medical S, Ribeirao Preto Medical S, St. Joseph's H, Medical C, St. Petersburg Academic U, Tayside Tissue B, University of D, University of Kansas Medical C, University of M, University of North Carolina at Chapel H, University of Pittsburgh School of M, University of Texas MDACC, Disease Working Group: Duke U, Memorial Sloan Kettering Cancer C, National Cancer I, University of Texas MDACC, Yonsei University College of M, Data Coordination Center CI, Project Team: National Institutes of $\mathrm{H}$ (2017) Integrated genomic characterization of oesophageal carcinoma. Nature 541(7636):169-175. https://doi.org/10.1038/natur e20805

4. Salem ME, Puccini A, Xiu J, Raghavan D, Lenz HJ, Korn WM, Shields AF, Philip PA, Marshall JL, Goldberg RM (2018) Comparative molecular analyses of esophageal squamous cell carcinoma, esophageal adenocarcinoma, and gastric adenocarcinoma. Oncologist 23(11):1319-1327. https://doi.org/10.1634/theoncolog ist.2018-0143

5. Mariette C, Balon JM, Piessen G, Fabre S, Van Seuningen I, Triboulet JP (2003) Pattern of recurrence following complete resection of esophageal carcinoma and factors predictive of recurrent disease. Cancer 97(7):1616-1623. https://doi.org/10.1002/cncr. 11228

6. Nakagawa S, Kanda T, Kosugi S, Ohashi M, Suzuki T, Hatakeyama K (2004) Recurrence pattern of squamous cell carcinoma of the thoracic esophagus after extended radical esophagectomy with three-field lymphadenectomy. J Am Coll Surg 198(2):205-211. https://doi.org/10.1016/j.jamcollsurg.2003.10.005

7. NCCN 2020 National Comprehensive Cancer Network. Clinical Practice Guidelines in Oncology. Esophageal and Esophagogastric Junction Cancers, Version 4. 2020-August 14, 2020. https:// www.nccn.org/professionals/physician_gls/pdf/esophageal.pdf. Accessed 3 Oct 2020

8. Burkart C, Bokemeyer C, Klump B, Pereira P, Teichmann R, Hartmann JT (2007) A phase II trial of weekly irinotecan in cisplatinrefractory esophageal cancer. Anticancer Res 27(4C):2845-2848

9. Iyer L, King CD, Whitington PF, Green MD, Roy SK, Tephly TR, Coffman BL, Ratain MJ (1998) Genetic predisposition to the metabolism of irinotecan (CPT-11). Role of uridine diphosphate glucuronosyltransferase isoform 1A1 in the glucuronidation of its active metabolite (SN-38) in human liver microsomes. J Clin Invest 101(4):847-854. https://doi.org/10.1172/JCI915

10. Kalra AV, Kim J, Klinz SG, Paz N, Cain J, Drummond DC, Nielsen UB, Fitzgerald JB (2014) Preclinical activity of nanoliposomal irinotecan is governed by tumor deposition and intratumor prodrug conversion. Cancer Res 74(23):7003-7013. https://doi. org/10.1158/0008-5472.CAN-14-0572

11. Drummond DC, Noble CO, Guo Z, Hong K, Park JW, Kirpotin DB (2006) Development of a highly active nanoliposomal irinotecan using a novel intraliposomal stabilization strategy. Cancer Res 66(6):3271-3277. https://doi.org/10.1158/0008-5472. CAN-05-4007

12. Kawato Y, Aonuma M, Hirota Y, Kuga H, Sato K (1991) Intracellular roles of $\mathrm{SN}-38$, a metabolite of the camptothecin derivative CPT-11, in the antitumor effect of CPT-11. Cancer Res 51(16):4187-4191
13. Peer D, Karp JM, Hong S, Farokhzad OC, Margalit R, Langer R (2007) Nanocarriers as an emerging platform for cancer therapy. Nat Nanotechnol 2(12):751-760. https://doi.org/10.1038/nnano. 2007.387

14. Simon R, Freidlin B, Rubinstein L, Arbuck SG, Collins J, Christian MC (1997) Accelerated titration designs for phase I clinical trials in oncology. J Natl Cancer Inst 89(15):1138-1147. https:// doi.org/10.1093/jnci/89.15.1138

15. Eisenhauer EA, Therasse P, Bogaerts J, Schwartz LH, Sargent D, Ford R, Dancey J, Arbuck S, Gwyther S, Mooney M, Rubinstein L, Shankar L, Dodd L, Kaplan R, Lacombe D, Verweij J (2009) New response evaluation criteria in solid tumours: revised RECIST guideline (version 1.1). Eur J Cancer 45(2):228-247. https://doi.org/10.1016/j.ejca.2008.10.026

16. Infante JR, Keedy VL, Jones SF, Zamboni WC, Chan E, Bendell JC, Lee W, Wu H, Ikeda S, Kodaira H, Rothenberg ML, Burris HA 3rd (2012) Phase I and pharmacokinetic study of IHL-305 (PEGylated liposomal irinotecan) in patients with advanced solid tumors. Cancer Chemother Pharmacol 70(5):699-705. https://doi.org/10.1007/s00280-012-1960-5

17. Chang TC, Shiah HS, Yang CH, Yeh KH, Cheng AL, Shen BN, Wang YW, Yeh CG, Chiang NJ, Chang JY, Chen LT (2015) Phase I study of nanoliposomal irinotecan (PEP02) in advanced solid tumor patients. Cancer Chemother Pharmacol 75(3):579586. https://doi.org/10.1007/s00280-014-2671-x

18. Blandizzi C, Danesi R, De Paolis B, Di Paolo A, Colucci R, Falcone A, Del Tacca M (2002) Cholinergic toxic syndrome by the anticancer drug irinotecan: acetylcholinesterase does not play a major role. Clin Pharmacol Ther 71(4):263-271. https:// doi.org/10.1067/mcp.2002.121909

19. Pitot HC, Goldberg RM, Reid JM, Sloan JA, Skaff PA, Erlichman C, Rubin J, Burch PA, Adjei AA, Alberts SA, Schaaf LJ, Elfring G, Miller LL (2000) Phase I dose-finding and pharmacokinetic trial of irinotecan hydrochloride (CPT-11) using a once-every-three-week dosing schedule for patients with advanced solid tumor malignancy. Clin Cancer Res 6(6):2236-2244

20. Kanbayashi Y, Ishikawa T, Kanazawa M, Nakajima Y, Tabuchi Y, Kawano R, Yoshioka T, Yoshida N, Hosokawa T, Takayama K, Taguchi T (2018) Predictive factors for the development of irinotecan-related cholinergic syndrome using ordered logistic regression analysis. Med Oncol 35(6):82. https://doi.org/10.1007/ s12032-018-1142-3

21. Harel M, Hyatt JL, Brumshtein B, Morton CL, Yoon KJ, Wadkins RM, Silman I, Sussman JL, Potter PM (2005) The crystal structure of the complex of the anticancer prodrug 7-ethyl-10-[4(1-piperidino)-1-piperidino]-carbonyloxycamptothecin (CPT11) with Torpedo californica acetylcholinesterase provides a molecular explanation for its cholinergic action. Mol Pharmacol 67(6):1874-1881. https://doi.org/10.1124/mol.104.009944

22. Blandizzi C, De Paolis B, Colucci R, Lazzeri G, Baschiera F, Del Tacca M (2001) Characterization of a novel mechanism accounting for the adverse cholinergic effects of the anticancer drug irinotecan. Br J Pharmacol 132(1):73-84. https://doi.org/10.1038/ sj.bjp.0703766

23. Rothenberg ML, Kuhn JG, Burris HA 3rd, Nelson J, Eckardt JR, Tristan-Morales M, Hilsenbeck SG, Weiss GR, Smith LS, Rodriguez GI et al (1993) Phase I and pharmacokinetic trial of weekly CPT-11. J Clin Oncol 11(11):2194-2204. https://doi.org/10.1200/ JCO.1993.11.11.2194

24. Zheng Y, Tu X, Zhao P, Jiang W, Liu L, Tong Z, Zhang H, Yan C, Fang W, Wang W (2018) A randomised phase II study of second-line XELIRI regimen versus irinotecan monotherapy in advanced biliary tract cancer patients progressed on gemcitabine and cisplatin. Br J Cancer 119(3):291-295. https://doi.org/10. 1038/s41416-018-0138-2 
25. Kato K, Cho BC, Takahashi M, Okada M, Lin CY, Chin K, Kadowaki S, Ahn MJ, Hamamoto Y, Doki Y, Yen CC, Kubota Y, Kim SB, Hsu CH, Holtved E, Xynos I, Kodani M, Kitagawa Y (2019) Nivolumab versus chemotherapy in patients with advanced oesophageal squamous cell carcinoma refractory or intolerant to previous chemotherapy (ATTRACTION-3): a multicentre, randomised, open-label, phase 3 trial. Lancet Oncol 20(11):15061517. https://doi.org/10.1016/S1470-2045(19)30626-6

26. Huang J, Xu J, Chen Y, Zhuang W, Zhang Y, Chen Z, Chen J, Zhang H, Niu Z, Fan Q, Lin L, Gu K, Liu Y, Ba Y, Miao Z, Jiang X, Zeng M, Chen J, Fu Z, Gan L, Wang J, Zhan X, Liu T, Li Z, Shen L, Shu Y, Zhang T, Yang Q, Zou J, Group ES (2020) Camrelizumab versus investigator's choice of chemotherapy as secondline therapy for advanced or metastatic oesophageal squamous cell carcinoma (ESCORT): a multicentre, randomised, open-label, phase 3 study. Lancet Oncol 21(6):832-842. https://doi.org/10. 1016/S1470-2045(20)30110-8

27. Kojima T, Shah MA, Muro K, Francois E, Adenis A, Hsu CH, Doi T, Moriwaki T, Kim SB, Lee SH, Bennouna J, Kato K, Shen L, Enzinger P, Qin SK, Ferreira P, Chen J, Girotto G, de la Fouchardiere C, Senellart H, Al-Rajabi R, Lordick F, Wang R, Suryawanshi S, Bhagia P, Kang SP, Metges JP, Investigators K (2020) Randomized phase III KEYNOTE-181 study of pembrolizumab versus chemotherapy in advanced esophageal cancer. J Clin Oncol 38(35):4138-4148. https://doi.org/10.1200/JCO.20. 01888

28. Kato K, Sun JM, Shah MA, Enzinger PC, Adenis A, Doi T, Kojima T, Metges JP, Li Z, Kim SB, Cho BCC, Mansoor W, Li SH, Sunpaweravong P, Maqueda MA, Goekkurt E, Liu Q, Shah S, Bhagia P, Shen L (2020) Pembrolizumab plus chemotherapy versus chemotherapy as first-line therapy in patients with advanced esophageal cancer: the phase 3 KEYNOTE-590 study. Ann Oncol. https://doi.org/10.1016/j.annonc.2020.08.2298

Publisher's Note Springer Nature remains neutral with regard to jurisdictional claims in published maps and institutional affiliations. 